\title{
SIMSCAPE Electrical Modelling of the IGBT with Parameter Optimization Using Genetic Algorithm
}

\author{
Mohamed Baghdadi $\mathbb{D}^{1},{ }^{1}$ Elmostafa Elwarraki, ${ }^{1}$ Naoual Mijlad, ${ }^{2}$ and Imane Ait Ayad $\mathbb{1}{ }^{1}$ \\ ${ }^{1}$ Department of Applied Physics, Faculty of Sciences and Technologies Cadi Ayyad University, Marrakech, Morocco \\ ${ }^{2}$ University Hassan II Ain Chock, Higher School of Technology, Casablanca, Morocco \\ Correspondence should be addressed to Mohamed Baghdadi; mohamed.baghdadi@ced.uca.ma
}

Received 23 November 2020; Revised 5 April 2021; Accepted 27 April 2021; Published 5 May 2021

Academic Editor: Herve Morel

Copyright (c) 2021 Mohamed Baghdadi et al. This is an open access article distributed under the Creative Commons Attribution License, which permits unrestricted use, distribution, and reproduction in any medium, provided the original work is properly cited.

\begin{abstract}
The concept introduced by MathWorks in the Simscape product is the link representation between the SIMSCAPE library components that correspond to physical connections transmitting power. In this paper, a power insulated-gate bipolar transistor (IGBT) model using MATLAB graphical software is reproduced. An electrical IGBT behavior model using the Simscape Electronics library components is developed and analyzed. This model is parameterized using the constructor datasheet to ensure a good representation of the dynamic and static IGBT behaviors. An extraction and optimization studies of the IGBT model parameters using a stochastic algorithm implemented in Matlab are presented. The proposed method is based on the Genetic Algorithm (GA) to perfectly extract and optimize the model parameters using the mathematical model circuit equations and the provided datasheet characteristics. A simulation in the Matlab/Simulink environment and a comparison with the experimental results for an IGBT device example are carried out to demonstrate the proposed model accuracy.
\end{abstract}

\section{Introduction}

For reducing design costs and increasing electronic power circuits' reliability, the modeling of power semiconductor devices becomes indispensable. The model must capture the dynamic and static behavior of the power semiconductor devices to ensure the safe global circuit operating area. The IGBT is one of the most important power semiconductor devices widely used in different power electronic applications.

In the literature, several IGBT models were developed at different complexity levels and for various uses. At this level, an accurate physical device description has been required in $[1,2]$. Hefner's model and a model list based on the Hefner work are examples of suitable mathematical models for circuit simulator implementation, which have proved to have a good accuracy and high reliability [3-5]. In [6, 7], the authors have proposed a complete and complex mathematical model using a complex equation for each model part, including the wide-base PNP section, the MOSFET channel section, capacities, the JFET effect, and the thermal effect. The difference between the analytical models is in the simplifications made in order to reduce the simulation time. To have more accurate results, numerical methods were employed to solve the $2 \mathrm{D}$ and $3 \mathrm{D}$ ambipolar diffusion equations. However, the numerical models' implementation requires special simulators. These model types are specially developed to optimize IGBT structure technologies [8].

For macromodels, they are used when high accuracy is not needed and the representation of some phenomena is ignored. Thus, the physical IGBT mechanisms are not considered in the models. Indeed, the fitted characteristics of the IGBT component are translated to an equivalent electrical circuit $[9,10]$.

The literature reported several works on the automatic extraction and optimization of IGBT model parameters. In [11], the authors developed the most suitable physical models and reference for the IGBT, and the IMPACT software has been elaborated to extract the IGBT model parameters. The IMPACT software has included five 
programs, which have extracted the 20 physical and structural parameters from the most recent version of the developed IGBT model. Each program has provided automatic algorithms to perform the extraction, using experimental measurements for each parameter set. The behavioral model of Oh and El Nokali implemented in Saber [12] has been reproduced by Kang et al. who have developed a new extraction algorithm to determine its parameters [13]. The algorithm is based on the availability of experimental switching loss data and the manufacturer-supplied characteristics, while the Matlab optimization toolbox is used to find model parameters automatically.

In [14], the IGBT model based on the finite-elements (FEM) physics has been implemented in the circuit simulator IsSPICE. The proposed extraction procedure is the numerical optimization algorithm called "Simulated Annealing" (SA). Its implementation is carried out in the Matlab programming environment, which allows a data exchange with IsSPICE.

This paper aims to model the IGBT device using the graphical Matlab software. The proposed electrical IGBT model is developed using Pspice $[15,16]$ and Saber simulators [17] in the previous contributions. The main contribution of this work is the automatic identification and optimization of the model parameters based on a stochastic algorithm. The nonlinear equations describing the IGBT model for each operating zone are rewritten as an optimization problem for their model parameters, which is solved using the Genetic Algorithm (GA) with the datasheet characteristics. The proposed technique is easily implemented in the Matlab environment, and the simulation results proved its high reliability. The rest of this paper is organized as follows: the electrical IGBT model is developed in Section 2, while Section 3 describes the model parameter optimization using GA. In Section 4, simulation results of the proposed model are discussed. Finally, conclusions are given in Section 5.

\section{IGBT Design}

2.1. Electrical IGBT Model. The insulated-gate bipolar transistor (IGBT) is a hybrid transistor, which includes the Metal Oxide Semiconductor Field Effect Transistor (MOSFET) at the input and the Bipolar Junction Transistor (TBJ) at the output. Consequently, the IGBT has low power control as an advantage of the MOSFET and presents the benefits of the TBJ which are low conduction losses and high-voltage handling [18]. The established model can predict the IGBT behavior for both dynamic and steady state. Besides, it can be easily parameterized using manufacturer data, and it represents an optimum compromise between accuracy and simulation complexity [17].

The static MOSFET model is constituted by

(i) $D_{G}$ : a diode which allows the passage from the blocked state to the on state.

(ii) $V_{\mathrm{TH}}$ : DC voltage source. It represents the threshold voltage.

(iii) $R_{\mathrm{GE}}$ : leakage gate-emitter resistance. (iv) $J_{\text {mos }}$ : a controlled current source which represents the drain current $\left(I_{\mathrm{mos}}\right)$.

(v) $R_{\mathrm{DN}}$ : drain resistance which characterizes the resistive aspect of the MOSFET channel.

(vi) $D_{\mathrm{DC}}$ : an antiparallel body diode which allows the passage from the linear zone to the saturated zone. It is represented by a binary resistance $R_{\mathrm{ONDC}} / R_{\mathrm{OFFDC}}$.

The MOSFET current is controlled by $V_{\mathrm{GE}}$ voltage, according to three cases:

(i) $V_{\mathrm{GE}}<V_{\mathrm{TH}}$ : the transistor is off, so the channel is not constructed

(ii) $V_{\mathrm{GE}}+V_{\mathrm{CE} 0}-V_{\mathrm{TH}}>V_{\mathrm{CE}}$ :

$$
I_{\text {mos }}=K_{p} \frac{\left(V_{\mathrm{GE}}-V_{\mathrm{TH}}\right) *\left(V_{\mathrm{CE}}-V_{\mathrm{CE} 0}\right)-\left(V_{\mathrm{CE}}-V_{\mathrm{CE} 0}\right)^{2} / 2}{\left(1+\theta\left(V_{\mathrm{GE}}-V_{\mathrm{TH}}\right)\right)}
$$

(iii) $V_{\mathrm{GE}}+V_{\mathrm{CE} 0}-V_{\mathrm{TH}}<V_{\mathrm{CE}}$ :

$$
I_{\operatorname{mos}}=\frac{K_{p}}{2} \frac{\left(V_{\mathrm{GE}}-V_{\mathrm{TH}}\right)^{2}}{\left(1+\theta\left(V_{\mathrm{GE}}-V_{\mathrm{TH}}\right)\right)},
$$

where $K_{p}$ is the transconductance and $\theta$ is the correction factor, which takes into consideration the transverse field in the MOSFET channel

For the bipolar part, the static TBJ model is composed by

(i) $D_{E}$ and $D_{P}$ : the base-emitter and base-collector junctions

(ii) $V_{\mathrm{CE} 0}$ : conduction threshold voltage of the baseemitter junction

(iii) $J_{\mathrm{PNP}}$ : a controlled current source which represents the current gain of the bipolar transistor

(iv) $\beta$ : the bipolar gain

2.2. Dynamic IGBT Model. To model the dynamic IGBT behavior, other elements are added to the static model. These elements include nonlinear terminal capacitances and the voltage-controlled current source that models the load responsible of the tail current $I_{\mathrm{C}}$ at the turn off IGBT state.

Constructors provide the measurement capacitors between two terminals by eliminating one or two capacitors. The input capacitance $\left(C_{\mathrm{IE}}\right)$, reverse transfer capacitance $\left(C_{\mathrm{RE}}\right)$, and output capacitance $\left(C_{\mathrm{OE}}\right)$ allow us to determine the interelectrode capacitance values $C_{G E}, C_{C E}$, and $C_{G C}$ using the following equations [19]:

$$
\left\{\begin{array} { l } 
{ C _ { \mathrm { IE } } = C _ { \mathrm { GE } } + C _ { \mathrm { GC } } } \\
{ C _ { \mathrm { OE } } = C _ { \mathrm { CE } } + C _ { \mathrm { GC } } } \\
{ C _ { \mathrm { RE } } = C _ { \mathrm { GC } } }
\end{array} \Longrightarrow \left\{\begin{array}{l}
C_{\mathrm{GE}}=C_{\mathrm{IE}}-C_{\mathrm{RE}} \\
C_{\mathrm{CE}}=C_{\mathrm{OE}}-C_{\mathrm{RE}} \\
C_{\mathrm{GC}}=C_{\mathrm{RE}}
\end{array}\right.\right.
$$

Figure 1 shows the evolution of $C_{\mathrm{GE}}, C_{\mathrm{CE}}$, and $C_{\mathrm{GC}}$ capacitances versus $V_{\mathrm{CE}}$. We note that the $\mathrm{C}_{\mathrm{GE}}$ capacity remains almost constant while the $C_{\mathrm{GC}}$ and $C_{\mathrm{CE}}$ capacities vary strongly nonlinear following the device operating area. 


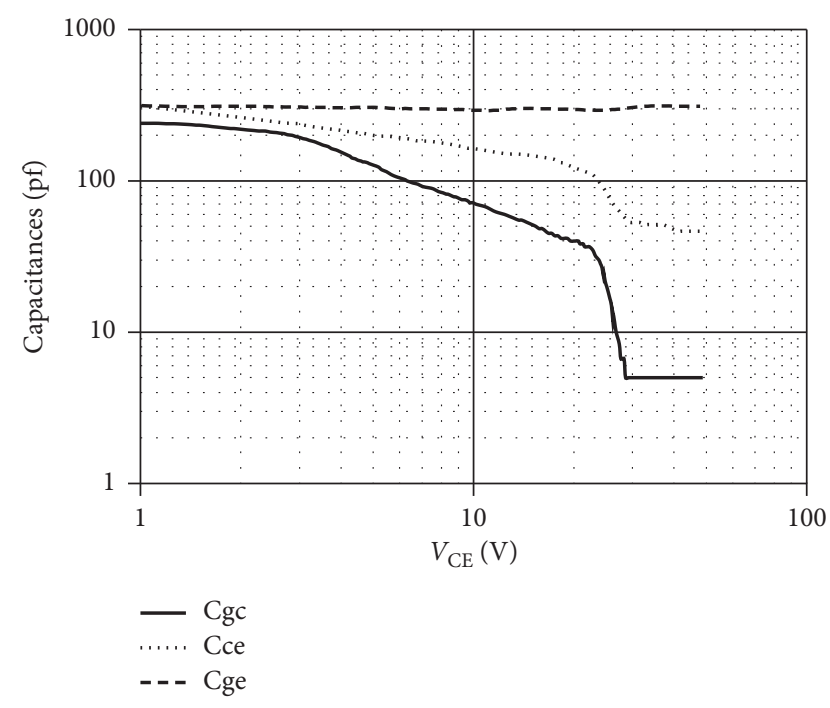

FIgURE 1: Curves of capacitances between terminals for the commercial IGBT (IRGBC20U) [18]. CGE capacity: its value is given by the system of equations (3).

These two capacities change values around the same threshold voltage $V_{\text {CET0 }}$.

$C_{\mathrm{GC}}$ capacity: this capacity can be constituted of two capacities in series: $C_{\mathrm{OXD}}$ oxide capacity that is fixed by the constructor and $C_{\mathrm{GCJ}}$ depletion capacity. In Figure 1, the $C_{\mathrm{GC}}$ capacity changes value from a certain $V_{\mathrm{CE}}$ threshold voltage $V_{\mathrm{CET}}$. We can use the diode model [16] to represent the nonlinearity of the $C_{\mathrm{GC}}$ capacity between two values $C_{\mathrm{GCmin}}$ and $C_{\mathrm{GCmax}}$ (Figure 2).

The $C_{\mathrm{GCmax}}$ capacity is represented by a voltage-controlled current source, with a gain given by the following expression:

$$
G_{\mathrm{GC}}=\frac{R_{\mathrm{onGC}} C_{\mathrm{GCmax}}}{L_{\mathrm{GC}}} .
$$

$C_{\mathrm{CE}}$ capacity: this capacity, as it seen in Figure 1, varies also as nonlinear. It is represented in the same manner as that for the $C_{\mathrm{GC}}$ capacity. The coupling coefficient $G_{\mathrm{CE}}$ is given by the following expression:

$$
G_{\mathrm{CE}}=\frac{R_{\mathrm{onGC}} C_{\mathrm{CEmax}}}{L_{\mathrm{GC}}}
$$

The bipolar part presents the responsible charge of the tail current $I_{C}$, and these charges can be represented only by the PNP base-emitter junction storing charges. Thereafter, these charges are modelled by a controlled source (Figure 3 ), whose expression is as follows:

$$
G_{t}=\frac{R_{\mathrm{onDE}} C_{\mathrm{BE}}}{L_{\mathrm{BE}}}
$$

where $C_{\mathrm{BE}}$ represents the bipolar part storing charges, with $C_{\mathrm{BE}} R_{\mathrm{onDE}}=t_{\mathrm{off}}$, where $t_{\mathrm{off}}$ is deduced from the device datasheets.

The studied IGBT model is shown in Figure 3.

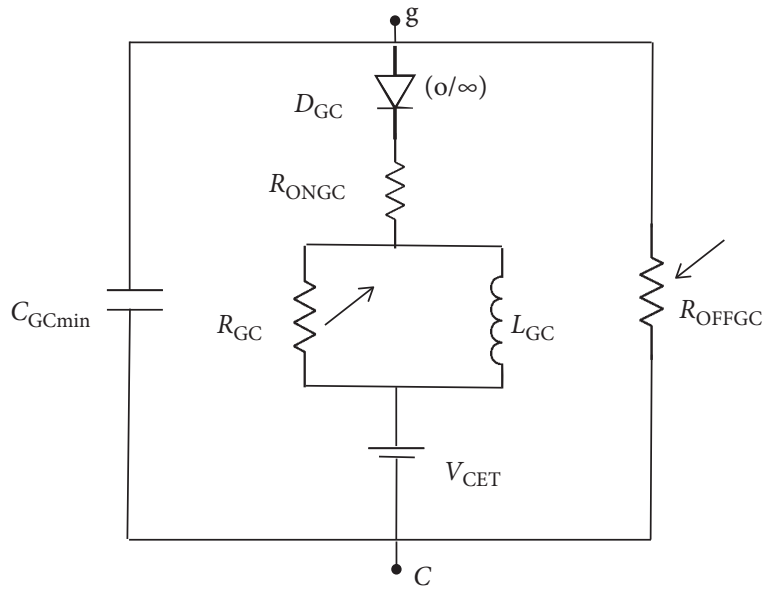

Figure 2: Model of the nonlinear $C_{\mathrm{GC}}$ capacity [15].

2.3. The IGBT Model Parameter Extraction. This section aims to develop and show the extraction method of the IGBT model parameters. To identify the model parameters in the static case, the main idea is to take out the circuit equations according to the diode states (Figure 4). Indeed, the diodes states define the different IGBT operation zones.

For the Ohmic zone, all diodes are turned on. The resolution of the equations system described the static model gives the expression of the $V_{\mathrm{CE}}$ voltage versus the $I_{C}$ current:

$$
\begin{aligned}
V_{\mathrm{CE}} & =V_{\mathrm{CE} 0}-\frac{\left(R_{\mathrm{ONDP}} * R_{\mathrm{ONDC}}\right)}{\left(R_{\mathrm{ONDP}}+R_{\mathrm{ONDC}}+R_{\mathrm{DN}}\right)} * I_{\mathrm{mos}} \\
& +\left(R_{\mathrm{ONDE}}+\frac{R_{\mathrm{ONDP}} *\left(R_{\mathrm{ONDC}}+R_{\mathrm{DN}}\right) *\left(1-G_{P} * R_{\mathrm{ONDE}}\right)}{\left(R_{\mathrm{ONDP}}+R_{\mathrm{ONDC}}+R_{\mathrm{DN}}\right)}\right) * I_{C} .
\end{aligned}
$$

For the quasisaturation zone, all the diodes are turned on except the $D_{P}$ diode. The expression of the $V_{\mathrm{CE}}$ voltage versus the $I_{C}$ current becomes

$$
\begin{aligned}
V_{\mathrm{CE}} & =V_{\mathrm{CE} 0}-\frac{\left(R_{\mathrm{ONDC}} * R_{\mathrm{OFFDP}}\right)}{\left(R_{\mathrm{ONDC}}+R_{\mathrm{OFFDP}}+R_{\mathrm{DN}}\right)} * I_{\mathrm{mos}} \\
& +\left(R_{\mathrm{ONDE}}+\frac{R_{\mathrm{OFFDP}} *\left(R_{\mathrm{ONDC}}+R_{\mathrm{DN}}\right) *\left(1-G_{P} * R_{\mathrm{ONDE}}\right)}{\left(R_{\mathrm{OFFDP}}+R_{\mathrm{ONDC}}+R_{\mathrm{DN}}\right)}\right) * I_{C} .
\end{aligned}
$$

For the saturation zone, it is characterized by the blocking diode $D_{\mathrm{DC}}$ of the MOSFET part and the blocking diode $D_{P}$ of the bipolar part. The expression of the $V_{\mathrm{CE}}$ voltage becomes

$$
\begin{aligned}
V_{\mathrm{CE}} & =V_{\mathrm{CE} 0}-\frac{\left(R_{\mathrm{OFFDC}} * R_{\mathrm{OFFDP}}\right)}{\left(R_{\mathrm{OFFDC}}+R_{\mathrm{OFFDP}}+R_{\mathrm{DN}}\right)} * I_{\mathrm{mos}} \\
& +\left(R_{\mathrm{ONDE}}+\frac{R_{\mathrm{OFFDP}} *\left(R_{\mathrm{OFFDC}}+R_{\mathrm{DN}}\right) *\left(1-G_{P} * R_{\mathrm{ONDE}}\right)}{\left(R_{\mathrm{OFFDP}}+R_{\mathrm{OFFDC}}+R_{\mathrm{DN}}\right)}\right) * I_{C} .
\end{aligned}
$$

The parameters of the studied model are deduced from the technical datasheet and the equations established in [15]. 


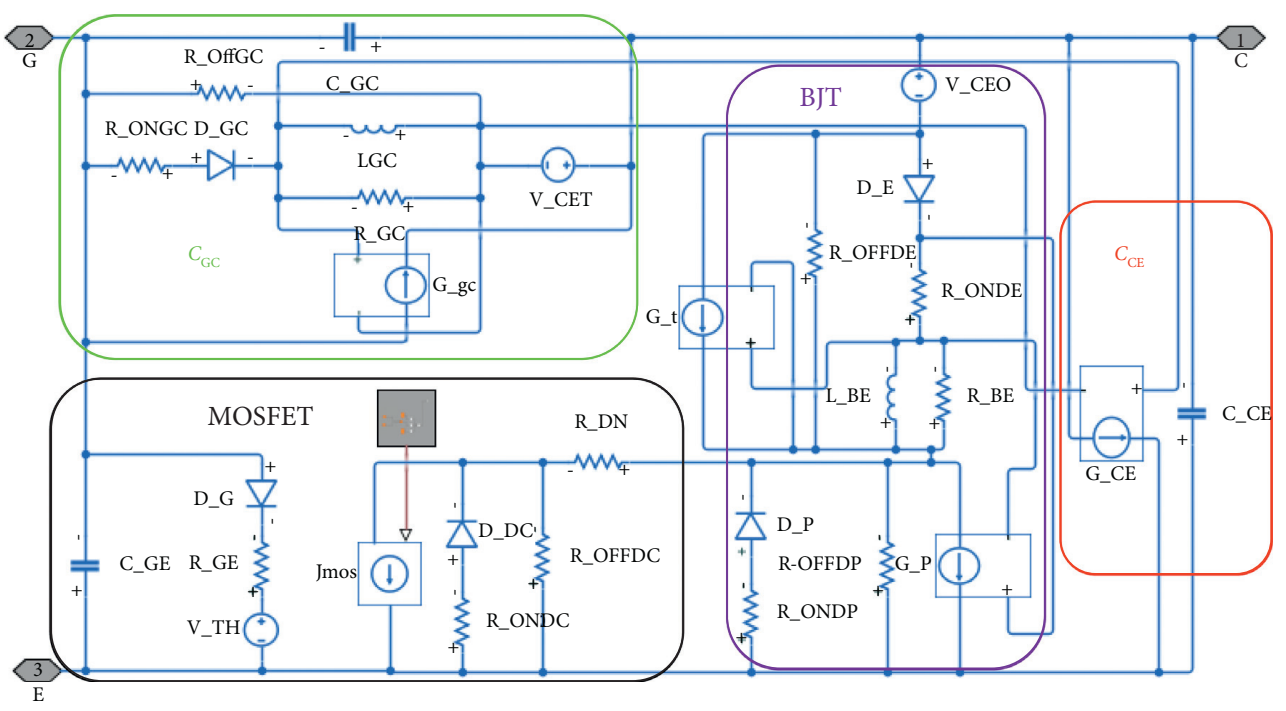

FIGURE 3: IGBT electrical model.

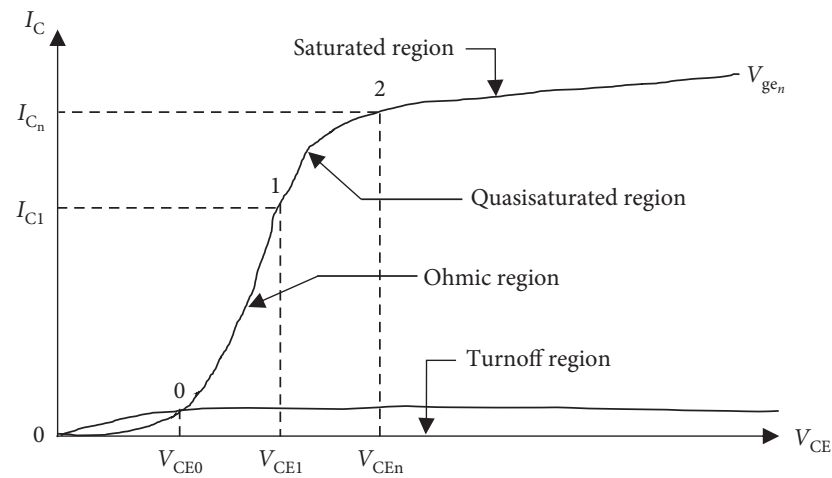

Figure 4: Decomposition of the static characteristic.

The IGBT parameters are recorded in Table 1 for the commercial IGBT (IRGBC20U).

\section{Optimization of the IGBT Model Parameters Using Genetic Algorithm}

3.1. Genetic Algorithm. In classical information treatment, the problems are solved in fixed ways before the introduction of heuristic methods using random processes. In fact, during the design phase of a system, it receives all the necessary data for its operating conditions as known at the time of its conception, which prevents its adaptation to unknown changing environmental conditions. Therefore, computer researchers are studying methods to allow systems to adapt spontaneously to new conditions. Hence, the heuristic and stochastic methods emerge such as evolutionary programming based on the Darwin theory. The theory of the species evolution is exposed under the external constraints influence, and the fixed systems' existence is rejected.

Among the evolutionary algorithms, the Genetic Algorithm (GA) is developed for optimization purposes. The genetic process is based on the natural selection theory and the evolution genetics. John Holland introduced the first formal model of genetic algorithms in 1976, which added an intelligence to a computer program with the crossing notion (genetic material exchange) and the mutation notion (genetic diversity source) [20]. Based on John Holland's work, several research studies have been developed.

Unlike the different optimization techniques, the GA is characterized by

(i) The use of an encoding describing the parameters

(ii) GA works on a population of points, instead of a single point

(iii) It uses only the function values under study

(iv) It uses probabilistic, not deterministic, transition rules

A genetic algorithm offers acceptable solutions for difficult problems in a reasonable calculation time. It tries to optimize the defined solution for a number of iterations and chooses the most optimized solution available for a problem at the program end. The initial creation of the population, the evaluation of the optimization condition (adaptation function or fitness function), selection, crossing, and mutation are the five fundamental functions of the algorithm.

Figure 5 shows the genetic algorithm diagram. The genetic algorithm process simulates the evolution of a population, which is subjected to a selection at each generation, and the recombination and mutation operators are applied. The selection based on the fitness function allows improving the population [20]. This algorithm does not require knowledge of the problem, which can be represented by a black box with inputs representing the variables and outputs representing the objective functions. Then, the algorithm manipulates the inputs in order to improve the outputs. 
TABLE 1: IGBT electrical parameters (IRGBC20U).

\begin{tabular}{|c|c|c|}
\hline & Parameters & Values \\
\hline Static parameters & $\begin{array}{c}V_{\mathrm{CEO} 0} \\
R_{\mathrm{ONDE}} \\
R_{\mathrm{DN}} \\
R_{\mathrm{ONDC}} \\
R_{\mathrm{OFFDC}} \\
R_{\mathrm{ONDP}} \\
R_{\mathrm{OFFDP}} \\
G_{P} \\
V_{\mathrm{TH}} \\
K_{P} \\
\Theta \\
R_{\mathrm{GE}} \\
\mathrm{B} \\
\end{array}$ & $\begin{array}{c}1.9 \mathrm{~V} \\
8.6610^{-2} \Omega \\
2.307310^{-3} \Omega \\
2.47910^{-1} \Omega \\
10^{8} \Omega \\
3.4210^{-3} \Omega \\
2.37310^{7} \Omega \\
1.154 \Omega^{-1} \\
5.3 \mathrm{~V} \\
1.766 \\
8.4710^{-2} \\
210^{8} \\
10^{-1}\end{array}$ \\
\hline Dynamic parameters & $\begin{array}{c}C_{\mathrm{GE}} \\
C_{\mathrm{GCmin}}, R_{\mathrm{GC}}, L_{\mathrm{GC}}, \text { RONGC } V_{C E T} \\
G_{\mathrm{GC}} \\
C_{\mathrm{CE}}, G_{\mathrm{CE}} \\
G_{t}\end{array}$ & $\begin{array}{c}310-10 \mathrm{~F} \\
610^{-12} \mathrm{~F}, 10^{-1} \Omega, 10^{-10} \mathrm{H}, 10^{3} \Omega, 20 \mathrm{~V} \\
2.4410^{-3} \mathrm{~F} \\
5.910^{-13} \mathrm{~F}, 3.0610^{3} \Omega^{-1} \\
910 \Omega^{-1}\end{array}$ \\
\hline
\end{tabular}

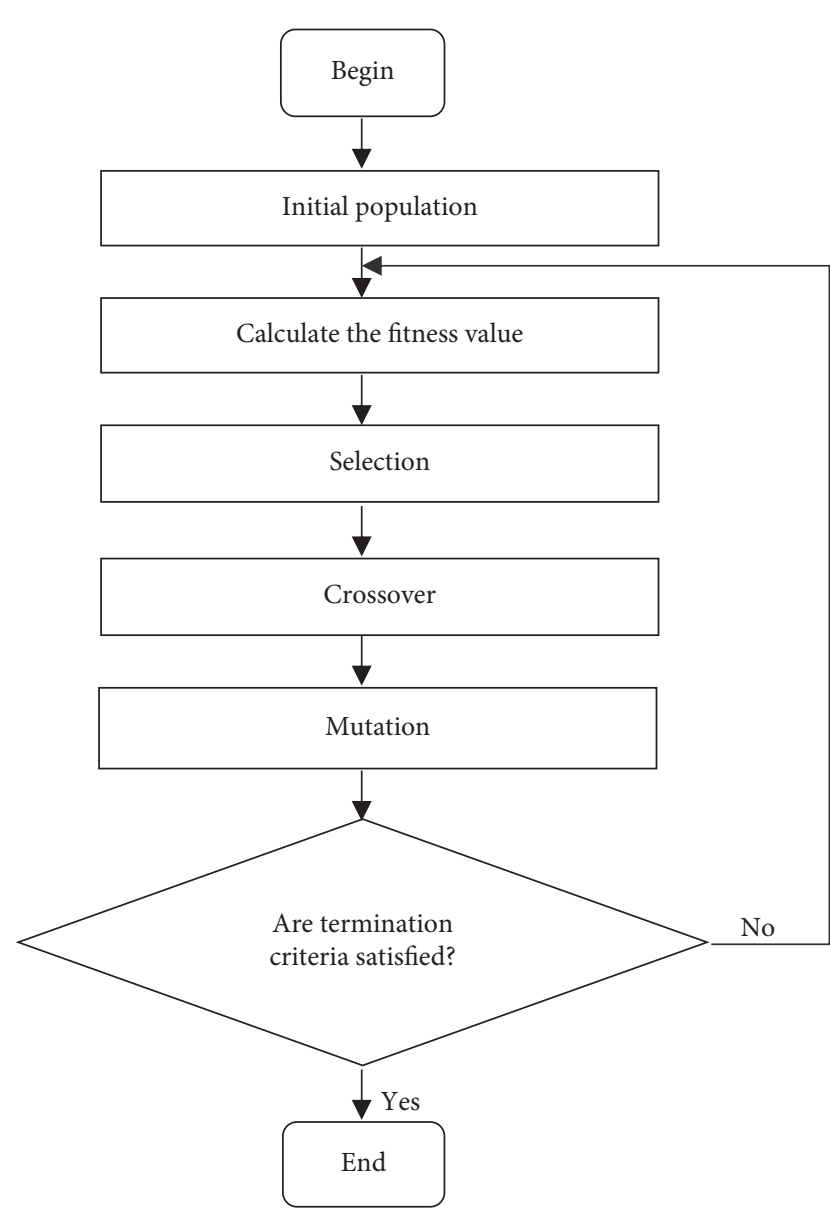

FIGURE 5: Flowchart of the standard genetic algorithm (GA).

3.2. Model Parameter Optimization Using GA. The GA is a robust stochastic process that provides accurate solutions to the optimization problem. Different domains of power electronic field have been benefited from the exploitation of GA. However, for optimization of semiconductors models, the GA method has been rarely used [21, 22].

Genetic algorithms belong to the evolutionary algorithms. They are based on natural selection principle and heredity. This concept is applied to an initial population of potential solutions $P(t)$ that gives several generations. For each generation, all individuals are evaluated according to an objective function [20]. Figure 6 shows the basic GA algorithm program.

In order to optimize the static parameters of the IGBT model, an approach is used to combine the manual extraction method that allows obtaining a first estimation of the set of parameters and then using the numerical optimization by the GA to extract the optimal set of parameters using the Toolbox "GA" in MATLAB [23].

The flowchart of the proposed optimization approach is presented in Figure 7.

The fitness function measures the error between manufacturer and simulated data. The used fitness function, for parameter evaluation, is defined by the sum of the deviation between the equation representing the manufacturer's curve (desired value) and the equation of the curve containing the model parameters (calculated values). The fitness function is given as follows:

$$
F_{\text {fitness }}=\sum\left(I_{\text {Cdata }}-I_{\mathrm{CM}}\right)^{2},
$$

where $I_{\text {Cdata }}$ is the value of the desired current given by the manufacturer and $I_{\mathrm{CM}}$ is the current value calculated by the simulated model.

Following that mentioned above, two programs are developed on MATLAB; the first one will use the mathematical equation of the transfer characteristic with equations (1) and (2) to optimize the coefficients $K_{P}, V_{\mathrm{TH}}, \theta$, and the bipolar gain $\beta$. The second program will use equations (7)-(9) with the mathematical equation of the output 


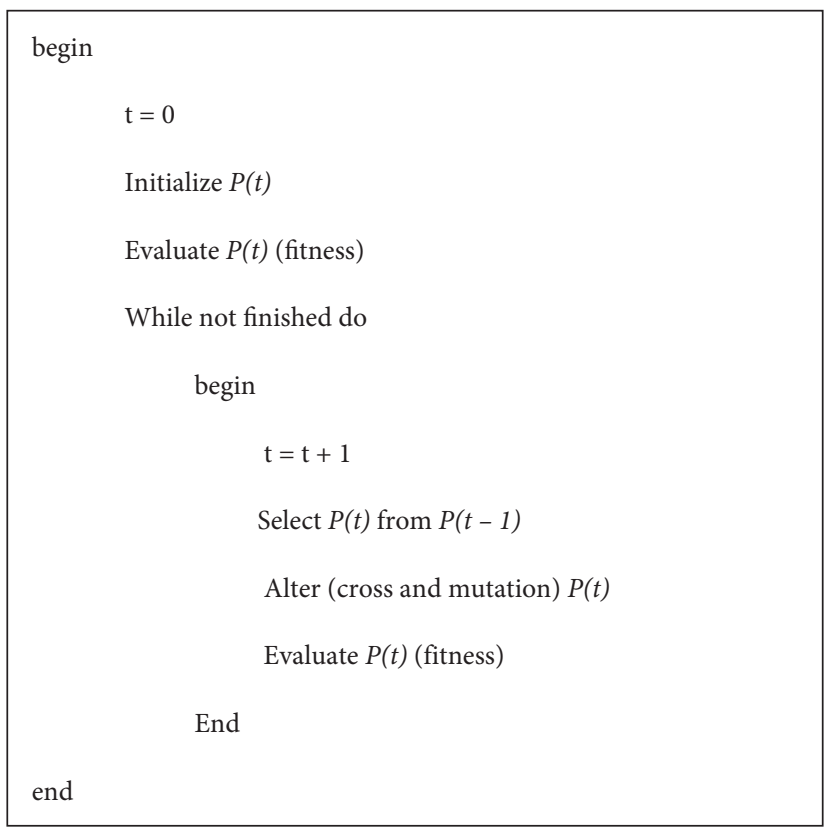

Figure 6: A simple genetic algorithm.

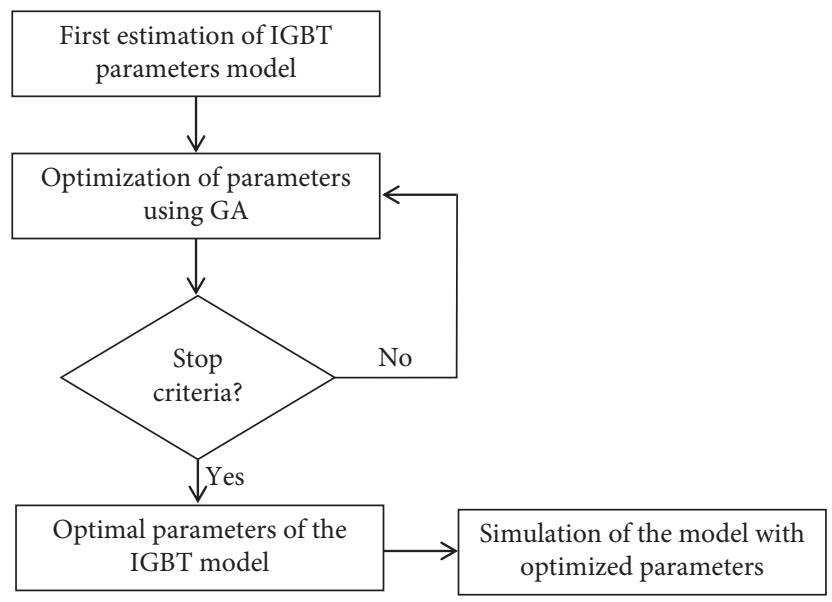

FIGURE 7: Organizational chart of the optimization approach applied to the IGBT model.

characteristic to calculate the other parameters $V_{\mathrm{CE} 0}, R_{\mathrm{ONDE}}$, $R_{\mathrm{DN}}, R_{\mathrm{ONDC}}, R_{\mathrm{OFFDC}}, R_{\mathrm{ONDP}}, R_{\mathrm{OFFDP}}$, and $G_{P}$.

The parameter values obtained by optimization and GA numerical information (data points and steps) are summarized in Tables 2 and 3, respectively.

Figure 8 shows the optimization results obtained for the two studied cases. A good convergence of the solution giving by the genetic algorithm in the treated cases can be seen.

The comparison between the obtained results and the simulation results without optimization is illustrated in Figure 9. The improvement brought by the model in the reproduction of the output and transfer characteristics in the saturation regime is shown. The error between the curves obtained by optimization and the curves given by the manufacturer is low.
TABLE 2: IGBT electrical parameters (IRGBC20U).

\begin{tabular}{lc}
\hline Parameters & Values \\
\hline$V_{\mathrm{CE0}}$ & $1.6119 \mathrm{~V}$ \\
$R_{\mathrm{ONDE}}$ & $8.0410^{-2} \Omega$ \\
$R_{\mathrm{DN}}$ & $2.010^{-3} \Omega$ \\
$R_{\mathrm{ONDC}}$ & $3.89310^{-1} \Omega$ \\
$R_{\mathrm{OFFDC}}$ & $4.69010^{6} \Omega$ \\
$R_{\mathrm{ONDP}}$ & $8.010^{-3} \Omega$ \\
$R_{\mathrm{OFFDP}}$ & $9.36110^{5} \Omega$ \\
$G_{P}$ & $1.3 .830 \Omega^{-1}$ \\
$V_{\mathrm{TH}}$ & $5.3 \mathrm{~V}$ \\
$K_{P}$ & 1.6166 \\
$\Theta$ & $8.1010^{-2}$ \\
$R_{\mathrm{GE}}$ & $210^{8}$ \\
$\mathrm{~B}$ & $1.34910^{-1}$ \\
\hline
\end{tabular}


TABLE 3: GA numerical information.

\begin{tabular}{lcr}
\hline & Data points & Steps (s) \\
\hline Transfer characteristic & 1451 & 2.283353 \\
Output characteristic & 851 & 0.748621 to 0.860017 \\
\hline
\end{tabular}

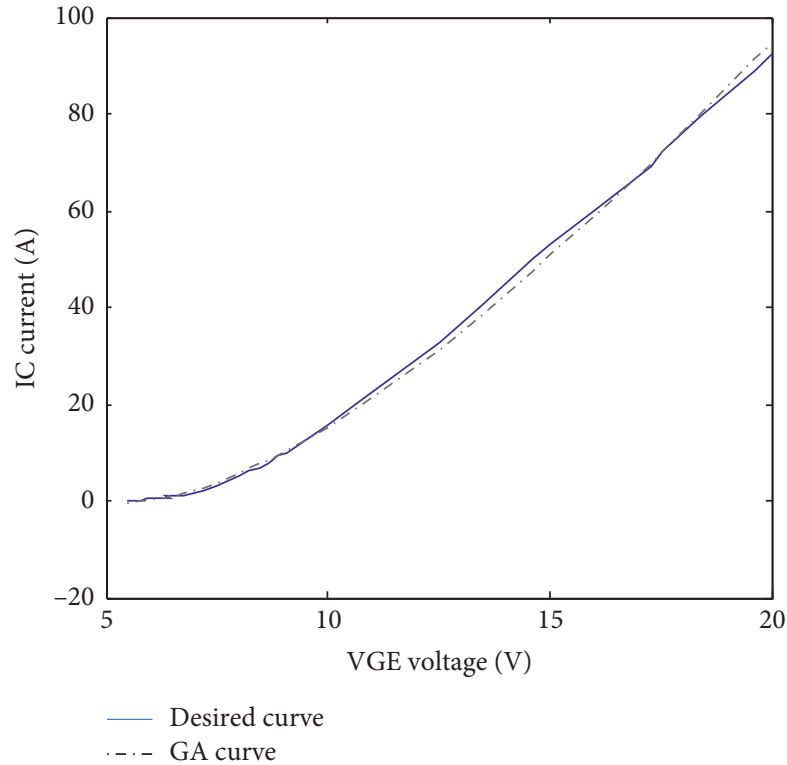

(a)

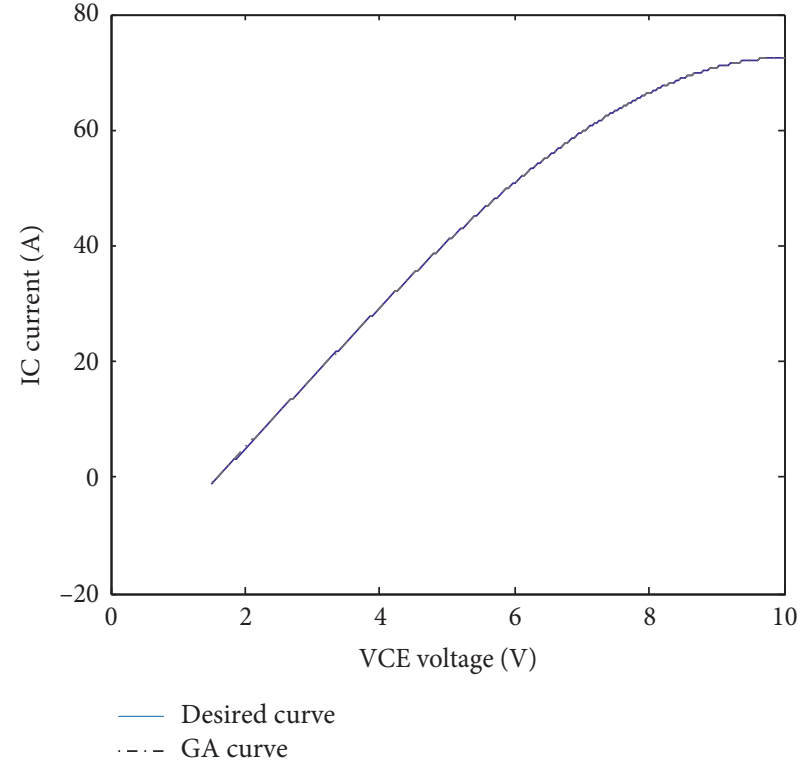

(b)

Figure 8: Convergence between manufacturer characteristics and GA curves giving optimal parameters. (a) Transfer characteristic. (b) Output characteristic.

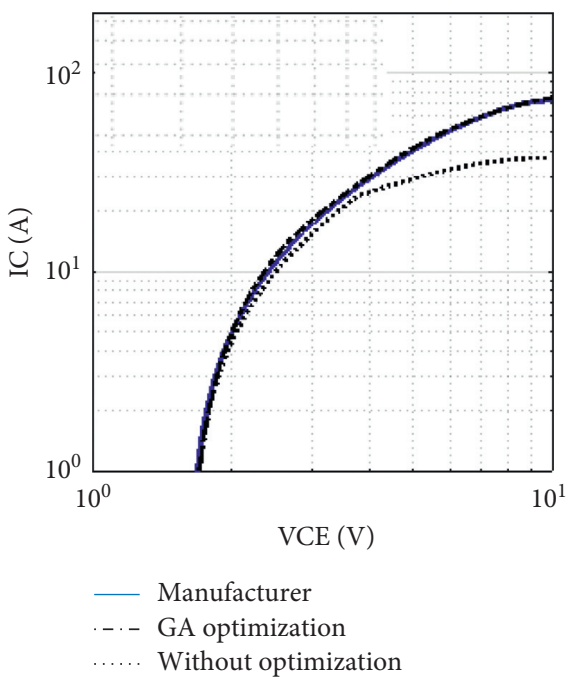

(a)

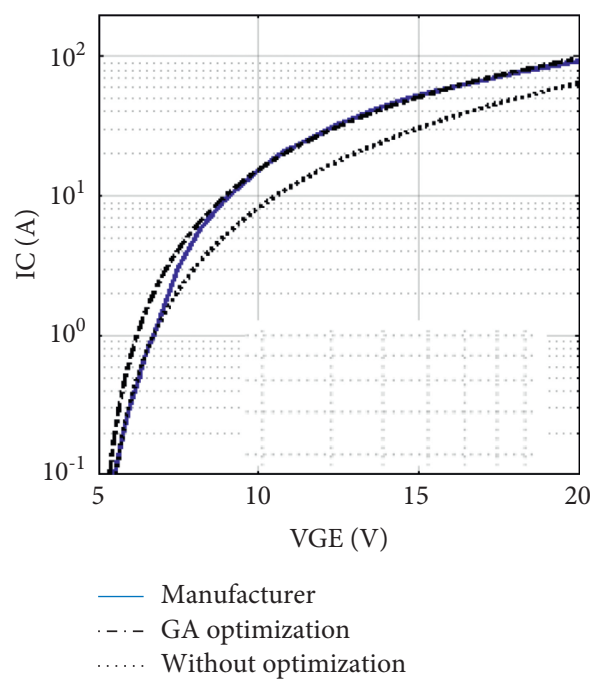

(b)

FIGURE 9: Simulation results after GA optimization. Transfer characteristic and output characteristic.

\section{Results and Discussion}

Figure 10 illustrates the experimental circuit used to perform the dynamic and static characteristics of the studied IGBT. The electrical IGBT environment is modelled by a wiring inductance $L_{P}$ and a wiring resistance $R_{P}$. A single parasitic inductance is sufficient to represent the total circuit inductances [24]. The IGBT driver causes the rise and fall times of the $V_{G}$ impulsion. This interaction between the control section and the device is also included in the test circuit simulation. 
LP

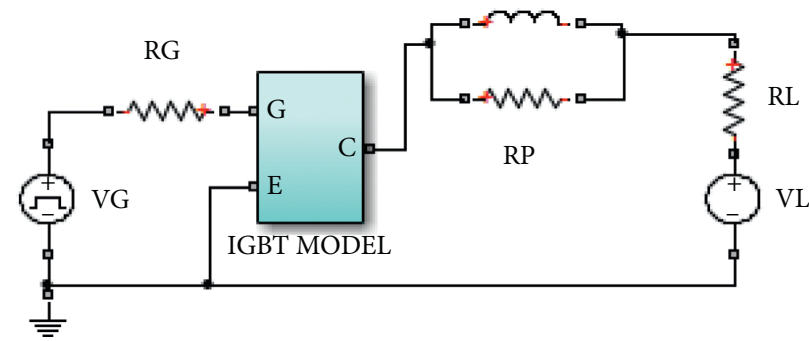

(a)

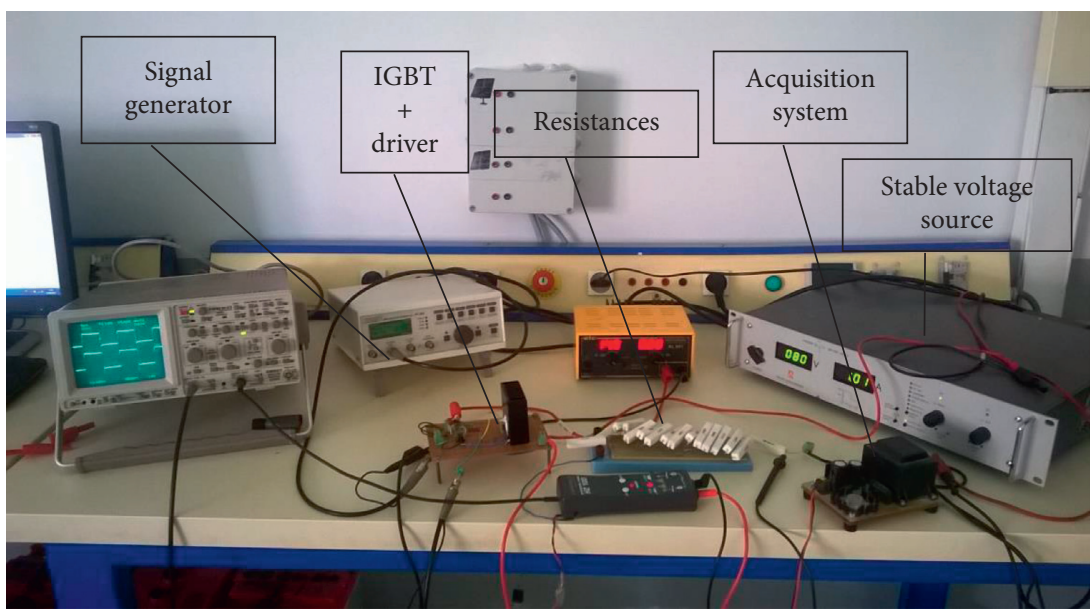

(b)

FIgure 10: (a) Simulated circuit. (b) Photography of the IGBT test bench.

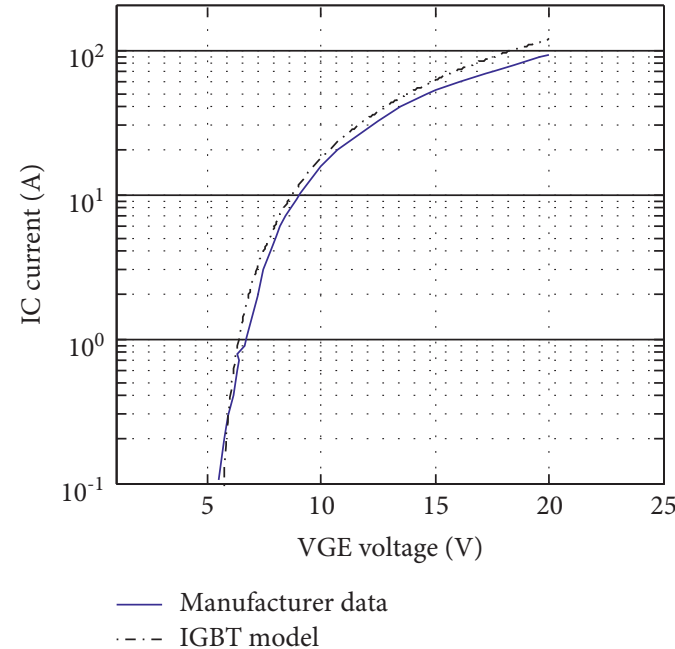

(a)

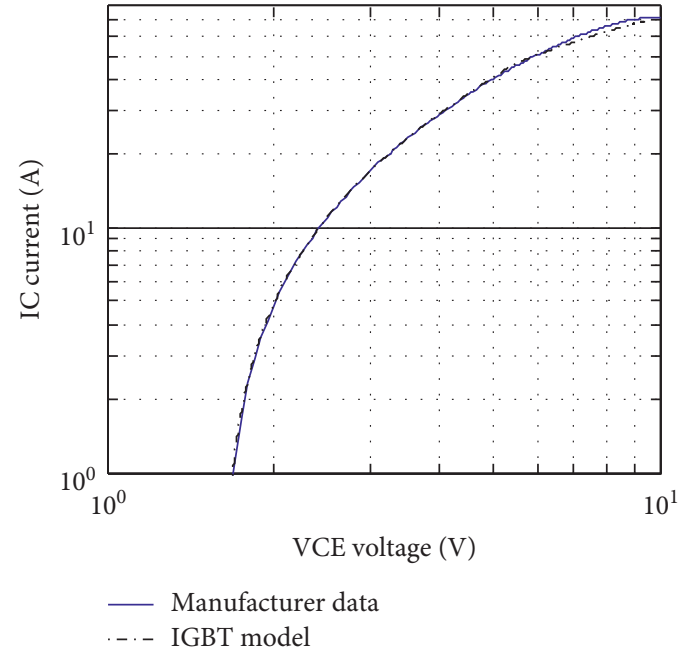

(b)

FIGURE 11: Experimental (manufacturer data) and simulated static characteristics of the commercial IGBT IRGBC20U, obtained for test conditions of the manufacturer. (a) Transfer characteristic. (b) Output characteristic.

4.1. Validation of the Static Behavior of the IGBT Model. To obtain the output characteristic, the simulation is performed as given in the manufacturer specifications in the following terms: the voltage $V_{\mathrm{GE}}$ is maintained at $15 \mathrm{~V}$, and the voltage $V_{\mathrm{CE}}$ changes linearly from $1 \mathrm{~V}$ to $10 \mathrm{~V}$ during $25 \mu \mathrm{s}$. Figure 11 shows experimental (manufacturer data) and simulated static characteristics of the commercial IGBT IRGBC20U, obtained for test conditions of the manufacturer: (a) transfer characteristic; (b) output characteristic. 


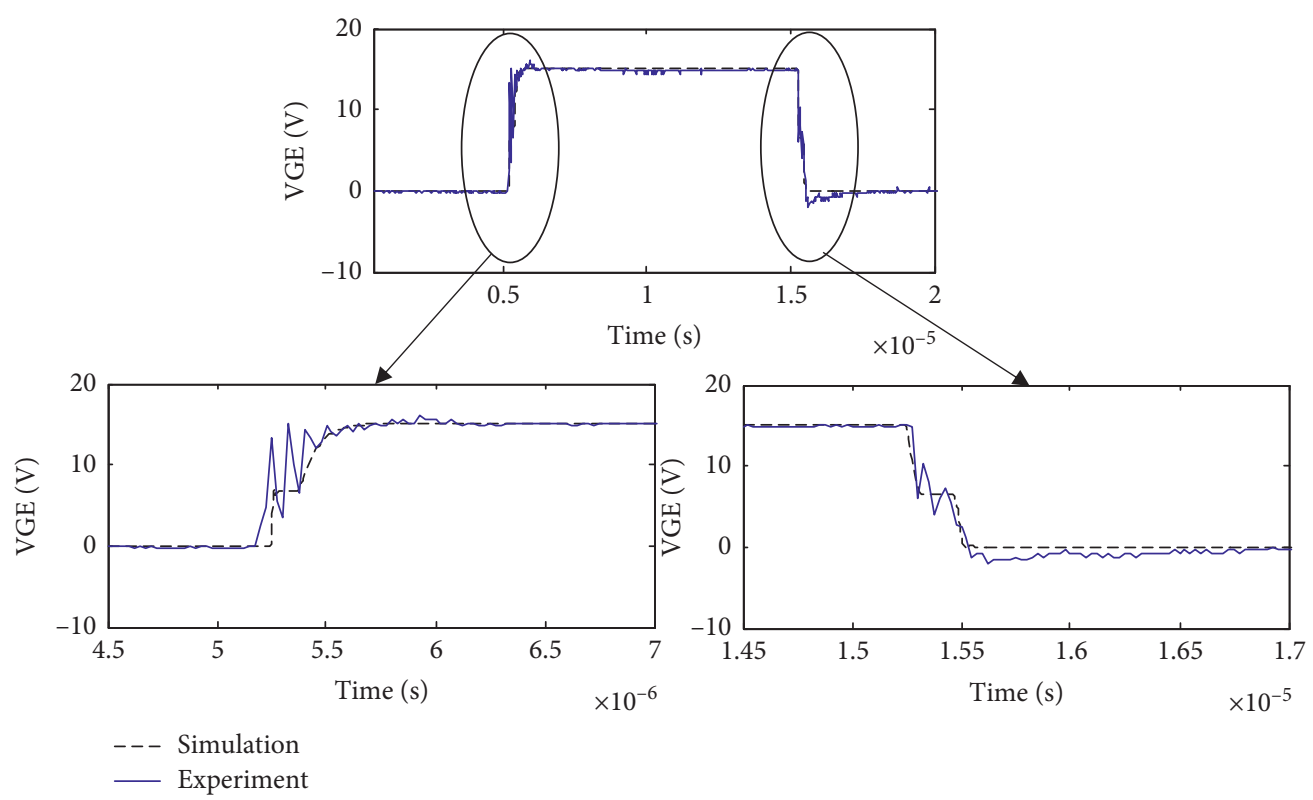

FIGURE 12: $\mathrm{V}_{\mathrm{GE}}$ waveforms of experimental and simulated dynamic IGBT (IRGBC20U) behaviour.

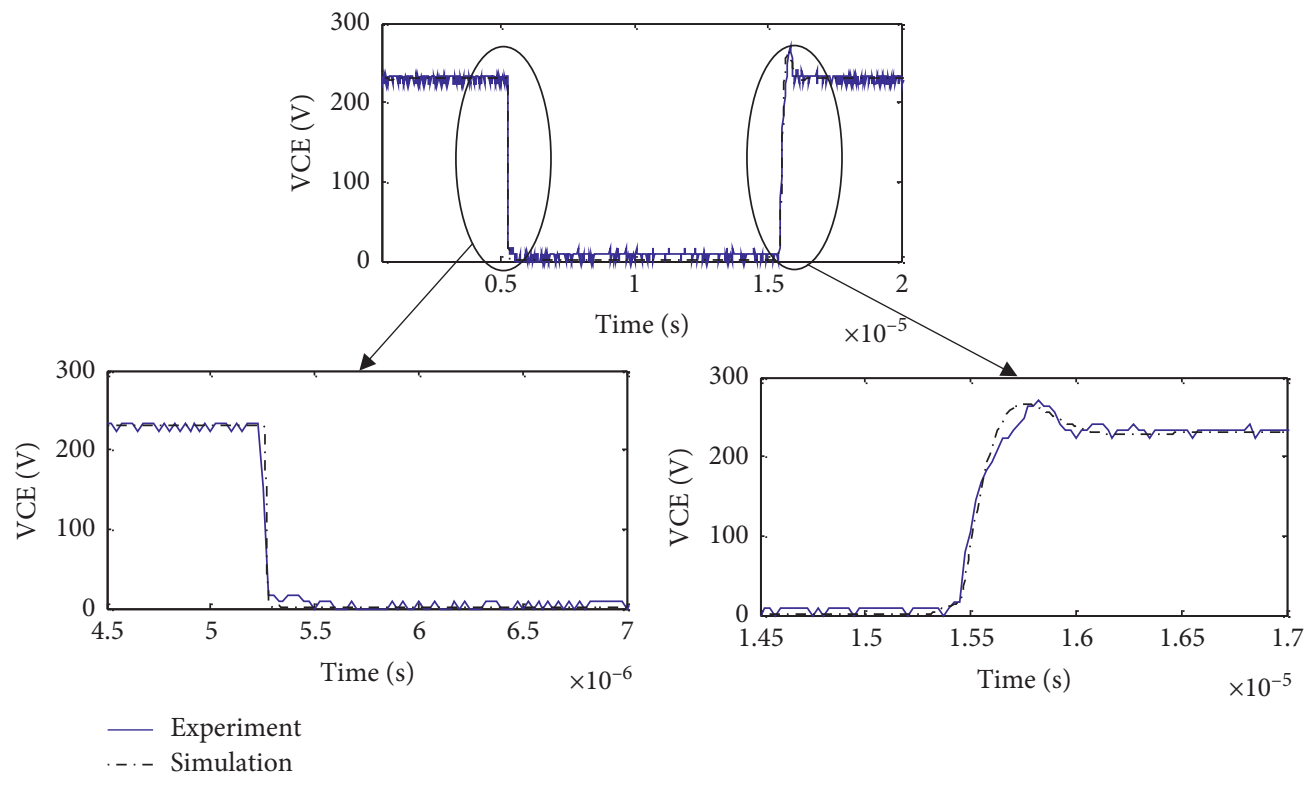

FIGURE 13: $V_{\text {CE }}$ waveforms of experimental and simulated dynamic IGBT (IRGBC20U) behavior.

To perform the transfer characteristic $I_{C}\left(V_{\mathrm{GE}}\right)$, the $V_{\mathrm{CE}}$ is fixed at $100 \mathrm{~V}$ while the $V_{\mathrm{GE}}$ varies from $5 \mathrm{~V}$ to $20 \mathrm{~V}$.

Figure 11 presents the manufacturer and simulation results of static characteristics. In the two curves, simulation results show good agreements compared to manufacturer data.

4.2. Validation of the Dynamic IGBT Model Behavior. The switching IGBT characteristics are affected by parasitic impedances, especially parasitic inductances. The $I_{\mathrm{C}}$ current decreases and $\mathrm{V}_{\mathrm{CE}}$ voltage increases at the turnoff switching phase owing to these parasitic inductances. According to Figures 12-14, it can be shown that the tail current $I_{\mathrm{C}}$ and the $\mathrm{V}_{\mathrm{CE}}$ voltage peak caused by these parasitic inductances increase the duration of the switching operation. Then, the switching losses at the turnoff phase are increased. The model predicts with a good accuracy all dynamic IGBT characteristics, namely, slowly decaying current at turn off (tailing phenomenon characteristic of IGBTs due to bipolar transistor part) and the Miller effect in the gate voltage waveforms. 


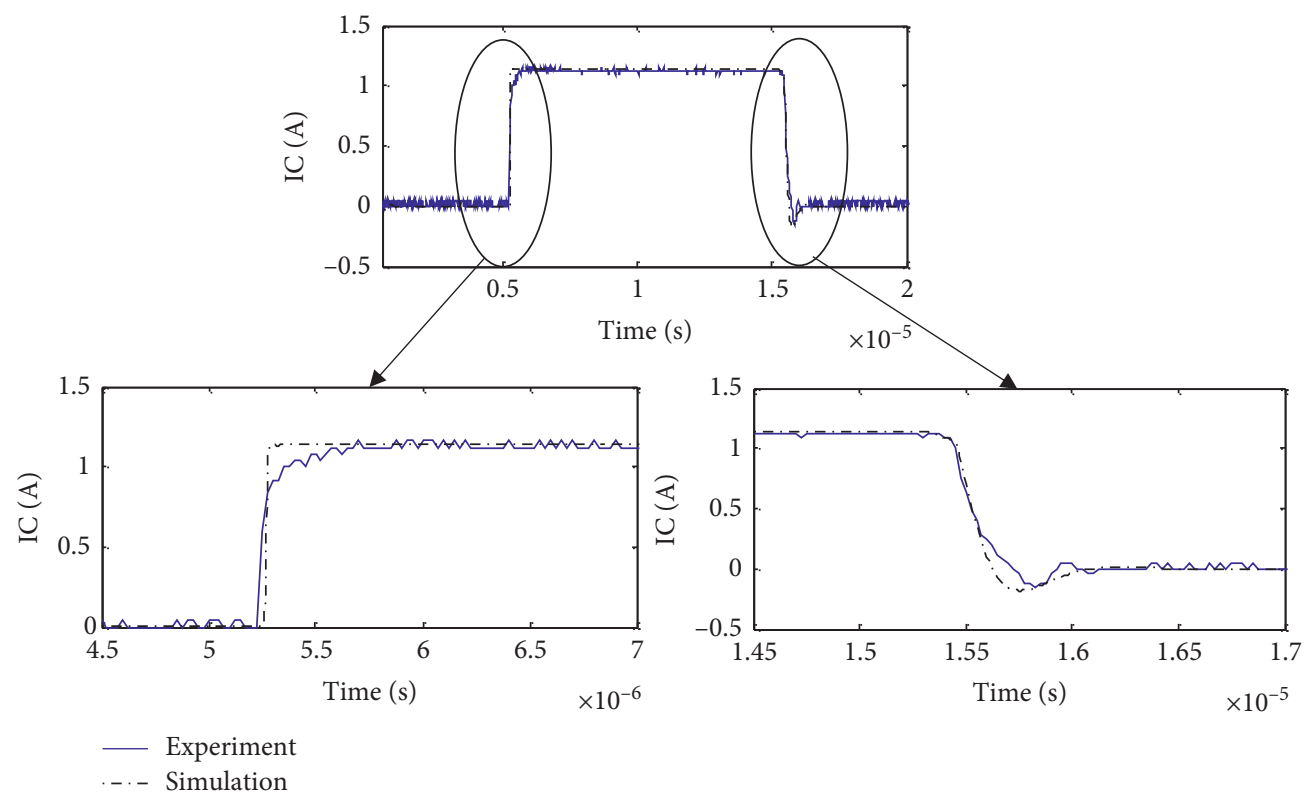

FIGURE 14: $I_{\mathrm{C}}$ waveforms of experimental and simulated dynamic IGBT (IRGBC20U) behaviour.

\section{Conclusions}

This study presents the electrical modeling of the IGBT using the graphical software of Matlab. Parameter optimization of the model based on a metaheuristic algorithm using GA was proposed. The established method used electrical equations of the model and measurement data provided by the manufacturer to perform the model parameter optimization. The method implementation was carried out using the Matlab/Simulink environment. Simulation of the electrical model showed a good precision and high reliability for the static and dynamic behavior of the used IGBT.

\section{Data Availability}

The data used to support the findings of this study have not been made available because they are confidential.

\section{Conflicts of Interest}

The authors declare that they have no conflicts of interest.

\section{References}

[1] K. Sheng, S. J. Finney, and B. W. Williams, "A new analytical IGBT model with improved electrical characteristics," IEEE Transactions on Power Electronics, vol. 14, no. 1, pp. 98-107, 1999.

[2] C. S. Mitter, A. R. Hefner, D. Y. Chen et al., "Insulated gate bipolar transistor (IGBT) modeling using IG-SPICE," IEEE Transactions on Industry Applications, vol. 30, no. no1, pp. 24-33, 1994.

[3] A. R. Hefner, "Analytical modeling of device-circuit interactions for the power insulated gate bipolar transistor (IGBT)," IEEE Transactions on Industry Applications, vol. 26, no. 6, pp. 995-1005, 1990.

[4] A. R. Hefner and D. L. Blackburn, "An analytical model for the steady-state and transient characteristics of the power insulated-gate bipolar transistor," Solid-State Electronics, vol. 31, no. 10, pp. 1513-1532, 1988.

[5] A. R. Hefner and D. M. Diebolt, "An experimentally verified IGBT model implemented in the Saber circuit simulator," in Proceedings of the PESC '91 Record 22nd Annual IEEE Power Electronics Specialists Conference, pp. 10-19, Cambridge, MA, USA, June 1991.

[6] B. Fatemizadeh and D. Silber, "A versatile electrical model for IGBT including thermal effects," in Proceedings of the IEEE Power Electronics Specialist Conference-PESC '93, pp. 85-92, Seattle, WA, USA, June 1993.

[7] B. Fatemizadeh, G. Tchouangue, and D. Silber, "A user-optimized electro-thermal IGBT model for power electronic circuit simulation in the circuit simulator ELDO," IEEE Applied Power Electronics Conference and Exposition, vol. 1, pp. 81-87, 1996.

[8] K. Sheng, F. Udrea, and G. A. J. Amaratunga, "Optimum carrier distribution of the IGBT," Solid-State Electronics, vol. 44, no. 9, pp. 1573-1583, 2000.

[9] Y. Tzou, Y. Yu, and L. Jun, “A practical SPICE macro model for the IGBT," IEEE International Conference on Industrial Electronics, Control, and Instrumentation, vol. 2, pp. 762-766, 1993.

[10] A. Monti, "A fuzzy-based black-box approach to IGBT modelling," in Proceedings of the Third IEEE International Conference on Electronics, Circuits, and Systems, pp. 11471150, Rhodes, Greece, August 1996.

[11] A. Hefner and S. Bouche, "Automated parameter extraction software for advanced IGBT modeling," in Proceedings of the 7th IEEE Workshop on Computers in Power Electronics, pp. 10-18, Troy, NY, USA, July 2000.

[12] H.-S. Oh and M. El Nokali, "A new IGBT behavioral model," Solid-State Electronics, vol. 45, no. 12, pp. 2069-2075, 2001.

[13] W. Kang, H. Ahn, and M. A. ElNokali, "A parameter extraction algorithm for an IGBT behavioral model," IEEE Transactions on Power Electronics, vol. 19, no. 6, pp. 13651371, 2004.

[14] R. Chibante, A. AraÚjo, and A. Carvalho, "Finite-element modeling and optimization-based parameter extraction 
algorithm for NPT-IGBTs," IEEE Transactions on Power Electronics, vol. 24, no. 5, pp. 1417-1427, 2009.

[15] E. Elwarraki and A. Sabir, "Behavioural and electrothermal modelling of the IGBT for circuits simulation," in Proceedings of the 4th IEEE International Conference on Electronics, Circuits and Systems, pp. 90-93, Marrakech, Morocco, December 2007.

[16] E. Elwarraki, Behavioural and electrothermal modelling of the bipolar devices adapted to the power function design, Ph.D. (Doctorat d'état), Faculty of Science Ben m'sik, Casablanca, Morocco, 2007.

[17] C. Alonso and T. A. Meynard, "Simulation of short-circuit phenomena in IGBT," in Proceedings of the IEEE Colloquium on Power Semiconductor Devices, pp. 101-105, London, UK, December 1994.

[18] N. Mijlad, E. Elwarraki, and A. Elbacha, "Implementation of a behavioral IGBT model in SIMULINK," in Proceedings of the International Conference on Electrical and Information Technologies (ICEIT'15), pp. 129-133, Marrakech, Morocco, March 2015.

[19] V. K. Khanna, Insulated Gate Bipolar Transistor IGBT Theory and Design, IEEE Press, Piscataway, NJ, USA, 2003.

[20] D. A. Coley and A. David, An Introduction to Genetic Algorithms for Scientists and Engineers, World Scientific Publishing Co Inc., Singapore, 1999.

[21] S. Busquets-monge, G. Soremekun, E. Hertz et al., "Design optimization of a boost power factor correction converter using genetic algorithms," in Proceedings of the Seventeenth Annual IEEE Applied Power Electronics Conference and Exposition, pp. 1177-1182, Dallas, TX, USA, March 2002.

[22] F. Alonge, F. D’Ippolito, and F. M. Raimondi, "Least squares and genetic algorithms for parameter identification of induction motors," Control Engineering Practice, vol. 9, no. 6, pp. 647-657, 2001.

[23] https://www.mathworks.com/help/gads/genetic-algorithm. html.

[24] Y. Shen, J. Jiang, Y. Xiong et al., "Parasitic inductance effects on the switching loss measurement of power semiconductor devices," in Proceedings of the IEEE International Symposium on Industrial Electronics, pp. 847-852, Montreal, QC, Canada, July 2006. 